\title{
Teoría sociológica, cultura moderna y emancipación: Un ejercicio inconcluso de auto-aclaración sociológica
} 3

\section{Cecilia Dockendorff}

Magíster en Antropología y Desarrollo, Universidad de Chile

Socióloga, Pontificia Universidad Católica de Chile

Directora Fundación Soles

cdocken@soles.cl

Resumen

El presente artículo presenta las características de la observación sociológica en torno a la modernidad y su relación con la cultura. La autora concluye que una observación sociológica de la cultura es necesaria para una teoría de la modernidad y de la intervención social.

\section{Abstract}

The present article presents the characteristics of the sociological observation around modernity and its relation with the culture. The author concludes that a sociological observation of the culture is necessary for a theory of modernity and the social intervention.

Palabras Clave: Teoría sociológica/ Modernidad/ Sistemas sociales/ Cultura/ Intervención social

\section{I ntroducción}

La arriesgada aventura que implica la pretensión de hacer teoría sociológica, en mi caso bajo la motivación derivada de una persistente y particular mirada a los problemas de la vida social contemporánea, exige un rigor que va más allá de adoptar un apellido sobre la etapa de la modernidad en curso o inclinarse por alguna de las teorías de la globalización. Implica preguntas y definiciones de fondo, esclarecer la lógica y la consistencia entre epistemología, metateoría, teoría y opciones metodológicas, amén de desentrañar valores, ética e ideología que se cuelan indefectiblemente tras las premisas teóricas adoptadas.

Tal esclarecimiento, un ejercicio de auto-aclaración sociológica, es lo que intentamos apenas comenzar en este ensayo, puesto que tanto por la dinámica propia de la evolución de la disciplina sociológica como por espacio, dicho ejercicio podrá avanzar sólo un trecho, quedando inevitablemente inconcluso.

En un primer punto nos asomamos al "reino de lo oculto", el mundo de lo tácito, lo latente, lo supuesto tras las opciones teóricas, la metateoría en el análisis de Ritzer (1988), el "hinterland" en palabras de Emmet (1994). En un segundo apartado intentamos establecer una conexión teórica entre ese mundo y el de la manifestación, los fenómenos, lo directamente observable, bajo la opción de considerar el ámbito de 
la vida cotidiana, dejando fuera de observación el de la institucionalidad. Por último, esbozaremos una posición ante la temática de la normatividad sociológica, exponiendo una propuesta específica de intervención social.

\section{El reino de lo implícito: Teoría sociológica, cultura, modernidad}

\section{Teoría sociológica}

Dos son aquí los ejes primarios de ubicación: el ámbito de la teoría general y el del papel de lo presupuesto. Respecto del primero, pretendemos ubicarnos en una sociología que desde su nacimiento buscó siempre un alto nivel de abstracción en sus formulaciones (Chernilo y Mascareño, 2005). Siguiendo la pretensión universalista tanto de los clásicos de la disciplina, Marx, Weber, Durkheim, Simmel y Parsons, como de contemporáneos como Habermas y Luhmann, nos mueve un afán por encontrar fundamentos universalistas que permitan encontrar la unidad de las diferencias observadas. Nuestra observación sin embargo, no puede pretender abarcar el fenómeno social en toda su extensión sino se dirige al plano semántico, el de la cultura, pero en un nivel de abstracción tal que permita reconocer una unidad, aun en la sociedad contemporánea caracterizada por un grado creciente de complejidad y diferenciación.

En cuanto al papel de lo presupuesto, concordamos con Alexander (1992) quien aborda la teoría -el corazón de la ciencia-, destacando que está afecta a elementos apriorísticos, es decir, a elementos no empíricos. Tradiciones que se dan por sentadas, influencias ideologías, modelos, distinciones sobre si la sociedad está en equilibrio o en conflicto, posiciones sobre la acción y sobre el orden social, son lo implícito destacado por Alexander; opciones epistemológicas y metafísicas lo que iluminan Ritzer (1988) y Emmet (1994). Nuestra atención se centrará en estos elementos apriorísticos de la teoría, pero no sólo de ésta, sino también en los que, a nuestro juicio, operan en la propia sociedad y son observables desde la concepción general de cultura que proponemos.

El gran territorio de la metafísica, la ontología y la epistemología que cada opción teórica trae consigo, es el primero en el que debemos tomar posición. Si usamos las distinciones que nos ofrecen Archer (1996) y Outhwaite (2006) quienes siguen los postulados filosóficos de Bashkar, deberíamos ubicarnos en alguna de las alternativas metateóricas que proponen: realismo empírico, idealismo trascendental o realismo trascendental. Su opción es el realismo trascendental, que distingue el dominio de lo empírico, lo actual, y lo real, esto es, las estructuras y mecanismos subyacentes. El principio esencial es la realidad de estructuras y de relaciones (en particular causales) en y entre estructuras. Desde esta perspectiva, el objeto de la ciencia son las estructuras, mecanismos y procesos de la realidad misma, estructuras y mecanismos que deben ser reales y no meras herramientas heurísticas (Outhwaite, 2006).

Contrariamente a la propuesta realista, nuestra opción es la perspectiva epistemológica constructivista, aquella que cuestiona el supuesto cartesiano de la separación sujeto-objeto y descarta, de ese modo, el realismo, considerado inescapable en la perspectiva antes expuesta. En efecto, contrariamente, las ya clásicas teorías cibernéticas y sobre el funcionamiento del cerebro y el sistema nervioso, entre las que se incluyen las de Heinz von Foerster (1990) y Humberto Maturana (1984) permiten sostener que la percepción se basa en una interpretación 
interna de estímulos no diferenciados, lo que significa que lo que se conoce del mundo es el resultado de procesos internos del sistema cognoscente. De este modo, el conocimiento no se basa en su correspondencia con la realidad externa, sino en las construcciones de un observador. Lo que éste interpreta como datos externos, no son sino distinciones, que, al comunicarlas como descripciones, aparecen como datos objetivos de la realidad (Arnold, 2004) ${ }^{1}$.

En el plano de la meta-teoría sociológica, la teoría de sistemas sociales de Luhmann adopta una epistemología concordante con las de la cibernética y la biología antes mencionadas. Siguiendo a Spencer-Brown, Luhmann (1998a) sustituye la diferenciación clásica sujeto-objeto por la de un observador que realiza distinciones. Este observador deja de ser descrito como un "sujeto" para ser conceptualizado como un sistema autónomo, autorreferencial. La epistemología luhmanniana enfrenta el proceso que produce conocimiento y construye realidades sociales distanciándose de las explicaciones o argumentos realistas que aún utiliza la tradición sociológica. Es más, Luhmann (1998a) sostiene que estas últimas permanecen entrampadas en lo que llama obstáculos epistemológicos. Entre ellos están la diferenciación sujeto-objeto, y el "prejuicio humanista", la creencia de que la sociedad está compuesta de seres humanos o de relaciones entre ellos.

Si ahora nos ubicamos en la perspectiva del diseño teórico en sociología, es decir, la forma en que se relacionan los componentes últimos de la realidad social, deberemos optar por una de las cuatro formas principales en que se construye la teoría: epifenominalismo (el orden social es un derivado natural de las propiedad de los elementos constituyentes de tal orden), elisionismo (la sociedad se constituye en una suerte de "punto medio" entre el individuo y las estructuras sociales), interpenetración (la sociedad emerge a partir de la contribución de sus elementos fundantes) y emergencia (la sociedad surge como un orden cualitativamente distinto, de los elementos que la fundan) (Chernilo, 2006). Según Archer (2005), en el debate actual sobre la relación agencia-estructura, el epifenimenalismo colectivista ha perdido adeptos en tanto los ha ganado el individualista, específicamente el individualismo metodológico. Resulta fácil una crítica al epifenimenalismo, en la medida que éste diluye las propiedades ya sea de los individuos o las estructuras al hacerlas derivar la una de la otra. Por su parte, la postura elisionista que intenta superar la derivación epifenomenalista, es también foco de críticas en la medida que la fusión de ambos componentes implica que cada cual pierde sus propiedades específicas. Es así como Archer (2005) se aparta de la teoría de la estructuración de Giddens, a la que acusa de elisionista, incapaz de separar analíticamente agencia de estructura, y propone un modelo que llama morfogenético con origen en una versión de teoría de sistemas. Por su parte, Outhwaite (2006) no cree que por la ruta sistémica se pueda dar solución al problema. A nuestro juicio, sin embargo, es justamente la perspectiva sistémica la que, por vía de la emergencia, logra mantener separados agencia de estructura sin que de ese modo pierdan ni impongan sus características.

Entre las posturas de interpenetración y de emergencia, representadas la primera por Parsons y Habermas y por Luhmann la segunda (Chernilo, 2006), creemos que adoptar las premisas luhmannianas permite enfrentar el dilema específico de la relación agencia-estructura de manera más satisfactoria, considerando la creciente complejidad de la sociedad contemporánea. En efecto, la emergencia permite entender el orden social como cualitativamente distinto de las propiedades de los individuos, considerados éstos sistemas síquicos autónomos relacionados a través del 
acoplamiento estructural con los sistemas sociales y la sociedad. Ambos, individuos y sistemas sociales mantienen su autonomía y ello permite a su vez superar los obstáculos epistemológicos que aun aquejan a la sociología. ${ }^{2}$ Creemos que la opción por la emergencia responde mejor a la extendida inquietud respecto a que la sociedad no es reductible a las acciones de los individuos pero tampoco existe independiente de éstos.

\section{Cultura}

Nuevamente son dos son las posiciones básicas que adoptamos, esta vez en relación a la cultura: la decisión de usar el concepto y el destacar el papel que desempeña lo implícito, lo presupuesto. Hemos afirmado que no sólo nos interesan los elementos apriorísticos de la teoría, sino también los que operan en la propia sociedad y forman parte de la concepción de cultura que proponemos.

El concepto de cultura no goza de aceptación entre los cultores de la teoría de sistemas sociales. Luhmann (1998b) opinaba que el concepto se ha expandido tanto que su amplitud ya casi impide establecer límites a lo que describe y resulta incompatible con la exactitud que requieren los conceptos científicos. A la extensión e imprecisión que ha adquirido el concepto, Luhmann agrega que se lo ha tratado como descripción de "algo" cuya existencia no se pone en duda, en lugar de entenderlo como una observación. En consecuencia, en Luhmann no encontramos una utilización del concepto como parte del andamiaje conceptual de la teoría, como tampoco un tratamiento de la función que la cultura puede tener a nivel del operar del sistema de la sociedad.

Por su parte, Mascareño (2007) sostiene que aquello que llamamos cultura está cada vez más destinado al abandono que corrieron conceptos de la física como el éter y el lambda, de no mediar la pregunta por la función de la cultura en un contexto de sistemas diferenciados operativamente clausurados. Según el autor, la cultura habría perdido su poder descriptivo y explicativo de las diferencias y semejanzas en una sociedad moderna y se habría vuelto "porosa".

Ante tales afirmaciones cabe preguntarse si se justifica el uso del concepto de cultura y si es posible restringir su amplitud y especificar su alcance. Creemos poder argumentar que sí; que la cultura no se ha vuelto porosa sino abstracta, y que mantiene una función en el contexto de sistemas diferenciados autónomos. Pero deberemos construir un concepto de cultura que muestre ser especialmente útil para describir la unidad de la sociedad contemporánea, puesto que ésta no se deja capturar por un concepto tradicional de cultura, en tanto es una sociedad policontextual en la que se da una simultaneidad de comunicaciones de enorme diversidad. Por otra parte, después de críticas como las antes expuestas, ya no es posible tratar la cultura como descripción de "algo" sino que debe entenderse como una observación, definido su alcance por el observador.

En tal sentido, sostenemos que cultura es el concepto que permite observar una unidad que, en la teoría de sistemas sociales aparece inarticulada en los conceptos de: horizonte de sentido, semántica, memoria sistémica, provisión de temas para la comunicación, expectativas, conocimiento y esquemas y guiones (Dockendorff, 2006a). Si establecemos una comparación con el lenguaje, que permite observar la unidad compuesta de fonemas, morfemas, sintagmas, etc. (Saussure, 1969), podemos 
observar que también cultura es un concepto que si se prescinde de él, los elementos que lo componen -o los conceptos que describen sus aspectos parciales-, pierden el sentido que los vincula y los integra en una realidad emergente que no es la mera sumatoria de éstos. Las semánticas sedimentadas de las que consta una cultura, constituyen una constelación coherente, esto es, mantienen una unidad de sentido entre sí.

Así como los árboles no dejan ver el bosque, creemos que el gran efecto evolutivo de la sociedad moderna en relación al proceso de diferenciación social, ha llevado a los sociólogos sistémicos a focalizar su reflexión en la diferenciación funcional de sistemas, lo que les ha impedido ver que si bien se ha perdido la hegemonía de un orden social centralizado en la sociedad contemporánea, no se ha perdido totalmente una unidad coherente de sentido. La teoría de sistemas sociales, al prescindir del concepto de cultura en lugar de especificar su alcance y función, se imposibilita para observar una unidad en la que la sociedad contemporánea continúa auto-describiéndose utilizando el concepto de cultura, mientras simultáneamente auto-observa su progresiva diferenciación. Sostenemos que es precisamente un concepto de cultura el que resulta útil para observar la unidad semántica que se da a pesar de la diferencia estructural (Dockendorff, 2006a).

Y es precisamente la teoría de sistemas sociales la que nos ofrece el contexto teórico adecuado para construir un concepto de cultura que supere los problemas que aquejan al concepto tradicional. Desde el punto de vista epistemológico, permite la construcción de un concepto de cultura que no necesita sustentarse en su correspondencia con una realidad externa, sino sólo en las construcciones de un observador. Asimismo, la teoría de sistemas sociales permite construir un concepto de cultura que, al evitar la pretensión de que la sociedad está compuesta por individuos, no necesita utilizar los conceptos de sujeto e intersubjetividad, amén de descartar toda referencia a la normatividad constitutiva del concepto tradicional. El concepto de cultura que se integra a la teoría de sistemas sociales no refleja un conjunto de valores y normas sino que se comprende como una unidad semántica en un contexto de diferenciación estructural (Dockendorff, 2006a).

Cultura apunta, de este modo, a una constelación de selecciones de sentido reestabilizadas en un nivel semántico que opera como una reducción de complejidad, en la medida que restringe el horizonte de sentido en el que se producen las comunicaciones sociales. Del orden emergente de la comunicación es posible observar, a su vez, la emergencia de la cultura, en la medida que se la conciba como comunicación reestabilizada. Ello implica una relación de recursividad entre comunicación y cultura, en tanto esta última es, a la vez, un resultado de la comunicación y un horizonte de sentido reducido que contiene a la propia comunicación. La cultura surge así como un resultado evolutivo de la comunicación y está, como la sociedad, constantemente evolucionando. La importancia que atribuimos a dicho concepto de cultura es que, junto al operar autónomo de los sistemas funcionalmente diferenciados y su dinámica de acoplamientos mutuos, va orientando la dirección de la evolución de la sociedad (Dockendorff, 2006a).

A dicha capacidad de orientación de la comunicación social, la hemos llamado función estructurante. En efecto, la semántica logra desarrollar dicha función estructurante en cuanto sufre un proceso de sedimentación. Es nuestra observación que las comunicaciones que se sedimentan van adquiriendo grados de sedimentación, en el 
sentido de estar más cerca o más lejos de expresarse en el lenguaje cotidiano; van, por decirlo así, cristalizando en capas sedimentadas a diversos grados de profundidad. Se conforma de este modo una constelación de comunicaciones sedimentadas, que hemos expuesto utilizando un modelo descriptivo que distingue un espacio semántico y una matriz cultural, componentes ambos de nuestro concepto de cultura. En el extremo de la máxima sedimentación distinguimos una constelación de semánticas sedimentadas en un grado de abstracción máximo, las que funcionan como esquemas de distinción y pueden ser descritas como supuestos o presupuestos respecto de categorías básicas referidas a la totalidad del mundo observado. A esta constelación de semánticas sedimentadas nos referimos como matriz cultural (Dockendorff, 2006a) ${ }^{3}$.

A pesar de estar extremadamente sedimentada y por ello lejos de la explicitación en el plano de la conciencia cotidiana, la matriz cultural ejerce una influencia decisiva en la vida cotidiana. Esto significa que el flujo comunicacional, una vez conformada una matriz cultural, se desenvuelve en una lógica o una dirección orientada por ella, la que se transforma en verdaderos nodos o puntos de arranque de comunicaciones que se van encadenando y que llevan implícitas, suponen, las selecciones estructurantes que conforman la matriz cultural (Dockendorff, 2006a) ${ }^{4}$. De este modo, la gran mayoría de las comunicaciones sociales son convergentes con la matriz cultural y son las divergentes (variaciones que se apartan de la matriz cultural), las que en ciertos períodos históricos conforman constelaciones que llegan a tener la capacidad de desplazar a las selecciones sedimentadas de la matriz cultural vigente, las que producen un cambio a nivel de la matriz cultural, lo que la sociedad observa y describe como cambios de épocas (Dockendorff, 2006b).

\section{Modernidad}

En relación a la modernidad destacaremos los mismos dos elementos con que hemos abordado nuestra opción de teoría sociológica y el concepto de cultura: un nivel general de abstracción y el papel de lo implícito.

Las primeras teorías sociológicas sobre la modernidad fueron maneras de capturar lo distintivo de lo que se llamó Modernidad: un modo de vida que emergió en Europa, con períodos de inicio variables según cada autor -entre los siglos XVI y XVIII- y con selecciones particulares de lo que consideran sus rasgos constitutivos. Los primeros autores fueron los clásicos como Saint-Simon, que destacaba el paso del antiguo régimen religioso a la sociedad industrial; de Tocqueville, el del antiguo régimen a la democracia; Marx y Engels con su distinción de sociedades pre-capitalistas y capitalistas; Toennies quien introduce la distinción que se ha hecho clásica entre Gemeinschaft y Gesellschaft; Weber desde las formas tradicionales de actividad económica al capitalismo y la organización racional de la vida; Durkheim de la solidaridad mecánica a la solidaridad orgánica y la compleja división del trabajo (Ourhwaite, 2006). Compartimos la visión de los clásicos en cuanto también destacamos un elemento central en la definición de lo que se entiende por modernidad, para nosotros el período de vigencia de su matriz cultural.

La evolución del pensamiento sobre la modernidad llega hasta nuestros días y, tal como acontece con el concepto de sociedad, cada perspectiva sociológica lleva implícita alguna concepción de la modernidad. Las recientes reflexiones sociológicas se dan en torno a la post modernidad y la globalización (Beck, 1998a; Bauman, 1999; Larraín 2005), incluyendo posturas como la de Albrow (en Outhwaite, 2006), quien 
sostiene que hemos ido más allá de la post-modernidad y hemos entrado en lo que llama la Edad o Era Global, en la que la sociedad se ha liberado de los límites del estado nación así como se liberó de la tradición irreflexiva. Aun reconociendo dichos efectos, nos distanciamos de las posturas que creen superada la modernidad, compartiendo con Habermas (1991) su visión de que ésta es aun un proyecto incompleto, con lo que no queremos decir que vaya alguna vez a completarse como tal.

Según Wagner (1997) la actual condición de la modernidad se caracteriza porque el aspecto normativo, es decir, el proyecto moderno, no ha sido traducido jamás a instituciones puras. Ello podría deberse a que la ambigüedad estaría presente desde la Ilustración en el proyecto moderno. En efecto, éste rechaza a un ser o principio impositivo sobre la libertad del individuo pero al mismo tiempo debe introducir al Otro (en potencial conflicto) lo que ha hecho en forma de Naturaleza Humana, Razón o Bien Común. Coincidimos con Wagner (1997) en cuanto a que la ambigüedad entre liberación y sometimiento es inherente al proyecto moderno, ${ }^{5}$ y sostenemos que dicha ambigüedad sólo podrá ser resuelta con un eventual reemplazo de la matriz cultural moderna.

Otros autores como Raymond Boudon, apuntan a las consecuencias inesperadas o los efectos perversos de la modernidad. Su observación es que los productos de la acción humana llegan a tener una existencia independiente y a dominar a sus creadores; lo que Weber analizaba como la racionalización y la burocracia y lo que Simmel llamaba la tragedia de la cultura (Outhwaite, 2006). Compartimos la perspectiva de los efectos inesperados de la modernidad pero encontramos para ello una explicación en la construcción del concepto de cultura que hemos esbozado, en tanto algunos de dichos efectos serían resultado de la escalada de comunicaciones que alcanza desarrollos extremos siguiendo los supuestos de la matriz cultural moderna, aun vigente.

Las perspectivas actuales incluyen también otros enfoques que nos resultan más afines en cuanto destacan las representaciones sociales, no sólo como reflejos de las relaciones sociales reales sino como constitutivas de dichas relaciones. Es así como Charles Taylor quien sigue a Castoriadis y Baczko, observa la modernidad occidental enfocado en lo que llama los imaginarios sociales modernos, es decir, las maneras en que la gente imagina su existencia social, sus expectativas y las imágenes que subyacen a tales expectativas. En este contexto, Taylor analiza cómo la gente llegó a pensar sobre sí misma como individuos, como individuos libres (Outhwaite, 2006). Para nosotros, la pregunta por el cómo la gente llegó a pensarse como individuos libres, contiene dos elementos centrales en nuestra perspectiva: la individualidad, como semántica constitutiva de la matriz cultural moderna, y "la gente", como expresión de la vida cotidiana y su relación con la capacidad estructurante de la matriz cultural.

Lejos de esta perspectiva está la mirada sistémica a la modernidad. Luhmann sostiene que son las características estructurales las que distinguen a la sociedad moderna, no las descripciones semánticas que, según él, terminan destacando rasgos unilaterales como "sociedad del riesgo" o "sociedad de la información", incapaces de dar cuenta de la complejidad de la sociedad (Luhmann, 1997: 18). Por su parte, Chernilo y Mascareño (2005) ven la modernidad en la sociedad mundial caracterizada por la diferenciación funcional y el cosmopolitismo. Creemos que nuestra perspectiva cultural puede agregar una dimensión que complejiza la visión de la modernidad y que posibilita 
observar en particular el ámbito de la vida cotidiana en la sociedad y la época modernas.

Es así como, desde nuestra perspectiva, y desde el punto de vista empírico, es posible observar la matriz cultural moderna. Al hablar de una "cultura de la modernidad" estamos haciendo una distinción muy gruesa, que permite múltiples otras distinciones al interior de ella. Estamos hablando de una época, que abarca un espacio amplio de tiempo y un territorio difícilmente delimitable que se extiende a lo que se ha denominado "occidente", aunque en rápida extensión a toda la sociedad mundial. La modernidad sería así una época que se caracteriza por la vigencia de una particular matriz cultural y que, si bien muestra una acelerada evolución observable en las comunicaciones sociales, las nuevas selecciones que puedan estar en proceso de sedimentación no han desplazado aun a aquellas que forman parte de su matriz cultural.

En cuanto a los contenidos de la matriz cultural moderna, la semántica de la individualidad es una de las selecciones estructurantes de alta abstracción y máxima generalidad que la conforman. Así operan también otras semánticas - materialidad del mundo, causalidad lineal, separación sujeto/objeto, linealidad temporal, entre otrasde manera similar a la individualidad ${ }^{6}$. Sostenemos que sobre ellas se alzan los fundamentos de nuestra cultura moderna y hacen posible distinguirla de la época que la precedió: la medieval ${ }^{7}$.

Es especialmente en la matriz cultural donde puede observarse la unidad de la sociedad moderna, y así como la matriz cultural moderna reemplazó a la matriz cultural medieval, la primera llegará a ser desplazada por alguna otra. Pero sólo la evolución de la sociedad, en la medida que encuentre las condiciones para reestabilizar comunicaciones que cuestionen las semánticas sedimentadas modernas, producirá dicho reemplazo e inaugurará una nueva época histórica con una matriz cultural nueva. Mientras ello no ocurra, la Modernidad cambiará de ropaje acorde con el despliegue de su propia lógica (su matriz cultural) y, aunque podrá dar origen a distinciones de etapas y nombres diversos, desde nuestra perspectiva sólo dejará de ser Modernidad cuando sea reemplazada la matriz cultural que la sostiene ${ }^{8}$.

\section{Lo oculto a la luz del sol: vida cotidiana, cultura moderna, sociedad contemporánea}

Hemos declarado la pretensión de establecer una conexión teórica entre el mundo de lo implícito, lo sedimentado a profundos niveles de abstracción, y el de los fenómenos, lo directamente observable. Es precisamente la gravitación que el nivel de lo implícito ejerce sobre la sociedad -en nuestro interés el ámbito de la vida cotidiana- que se justifica su distinción y análisis. Es sólo en función de estos rendimientos que logrará tener sentido una perspectiva como la propuesta, incluyendo su relevancia para eventuales iniciativas de intervención social.

\section{Vida cotidiana}

Nos enfrentamos aquí a problemas teóricos respecto de cómo distinguir y conceptualizar el ámbito de la vida cotidiana y la relación latencia-acción, sin recurrir a perspectivas fenomenológicas que descansan en los conceptos de intersubjetividad e internalización. Hemos optado por la teoría de sistemas sociales, cuyos fundamentos 
epistemológicos nos han permitido superar los problemas ontológicos que supone la separación sujeto-objeto, y resolver también el dilema de la relación agenciaestructura, por lo que a su interior deberemos encontrar un lugar para la vida cotidiana y una solución al problema de la internalización.

¿Cómo llegan hasta los sistemas psíquicos, que participan en la comunicación social, los supuestos de que consta la matriz cultural vigente? Luhmann reconoce que "ni siquiera disponemos de esbozos para una teoría empírica del funcionamiento recursivo, autorreferencial, de los sistemas de conciencia" (Luhamnn, 1998a: 274). No obstante, puede resolver el tema de la internalización y la socialización a partir de la noción de acoplamiento estructural que se produce entre los sistemas síquicos y los sistemas sociales. Ambos tipos de sistemas consisten de elementos que apenas emergen se desvanecen: ideas y comunicaciones. Ello los hace poder coincidir, de modo que las comunicaciones son a la vez eventos en las conciencias de los sistemas síquicos participantes, aunque se mantiene su estricta independencia operativa. De este modo, la socialización no puede entenderse como una simple transmisión de modelos de conformidad sino como una auto-socialización que contempla siempre las alternativas tanto de conformidad como de desviación (Luhmann, 1998a: Cap.12).

Es precisamente en relación a esta perspectiva que nos parece relevante la idea de que la emergencia de la cultura, como comunicación reestabilizada, logra desarrollar una función estructurante en cuanto sufre un proceso de sedimentación. Hemos afirmado que la matriz cultural se convierte en verdaderos nodos o puntos de arranque de comunicaciones que se van encadenando y que llevan implícitas las selecciones estructurantes que la conforman. De esta manera, en la medida que los sistemas síquicos se acoplan a las comunicaciones sociales, participan del flujo comunicacional orientado por la matriz cultural, en el que encuentran puntos de partida para nuevas comunicaciones, ya sean éstas convergentes o divergentes de la orientación recibida. Es precisamente la posibilidad de desviación, lo que hace a los sistemas síquicos la fuente de irritación hacia el cambio social, de llegar a ser reestabilizadas comunicaciones divergentes por la sociedad (Dockendorff, 2006b).

En cuanto a la vida cotidiana, no podemos recurrir a conceptos como mundo de la vida, en el que Habermas (1991) ubica el lugar de la racionalidad comunicativa, a diferencia del ámbito sistémico en el que primaría la racionalidad instrumental. Nos parece ficticia esta diferenciación si consideramos el punto de vista de los individuos, los que pueden utilizar ambos tipos de racionalidad ya sea en el mundo de la vida como a nivel de sistemas funcionales. Nos parece que la teoría de sistemas sociales puede ofrecer mejores posibilidades teóricas para la distinción de la vida cotidiana, por lo que deberemos encontrar su lugar al interior de ésta. Creemos poder hacerlo a partir de la diferenciación que establece dicha teoría entre sistemas sociales y sistemas interaccionales. Luhmann no desarrolló una teoría especial de los sistemas interaccionales pero los definió claramente: "los sistemas de interacción se forman cuando, para resolver a través de la comunicación el problema de la doble contingencia, se usa la presencia de personas" (Luhmann y De Georgi, 1993: 354) De este modo, a diferencia de los sistemas sociales que sólo requieren de comunicación para su formación, los sistemas interaccionales deben agregar a la comunicación, la presencia física de los individuos (sistemas síquicos acoplados a sistemas orgánicos).

La distinción entre sistemas funcionales y organizacionales por un lado, y sistemas interaccionales por otro, nos permite diferenciar entre el plano institucional y el de la 
vida cotidiana. Diremos así, que la vida cotidiana corresponde al ámbito de los sistemas interaccionales. Debemos reconocer, sin embargo, que se trata de una distinción analítica, puesto que en la sociedad ambos tipos de sistemas operan en referencia unos de otros (Luhmann, 1991: cap.10).

\section{La cultura moderna en la cotidianeidad de la sociedad contemporánea}

Son las consecuencias de la vigencia de la matriz cultural moderna para la vida cotidiana en la sociedad contemporánea lo que le otorga sentido a la teoría de la cultura y la identificación de la matriz cultural que hemos expuesto. A nuestro juicio, gran parte de los problemas del hombre contemporáneo dice relación con la evolución de la sociedad -entendida como las comunicaciones sociales- orientada por la matriz cultural moderna. Obviamente no pretendemos desconocer los problemas estructurales derivados, entre otros, de los efectos de la diferenciación funcional. Sin desconocer la profundidad del impacto de la modernidad tardía y en especial de la globalización sobre la sociedad contemporánea, creemos que la perspectiva que destaca los efectos acumulativos de semánticas sedimentadas por largo tiempo nos parece ausente del debate en torno a la modernidad $y$, a nuestro juicio, puede constituir una mirada complementaria que complejiza el diagnóstico de los problemas contemporáneos.

Hemos afirmado que la importancia que atribuimos a la vigencia de la matriz cultural moderna dice relación con su poder estructurante. En efecto, el desenvolvimiento de las comunicaciones sociales llega a ser una escalada lógica, en la medida que va logrando objetivos o alcanzando estadios que se transforman en puntos de partida para nuevas comunicaciones. De esta manera, la matriz cultural moderna, por general y abstractos que sean sus contenidos, está presente en cada nueva instancia de comunicación social $y$, dado que dicha matriz cultural moderna se ha mantenido vigente por un período aproximado de 400 años (Dockendorff, 2002), sus desarrollos han adquirido niveles tan extremos que son observados hoy como críticos (Giddens, 1993; Beck, 1998b).

Algunos de los problemas contemporáneos que desde otras perspectivas se analizan como resultado de fenómenos modernos como la globalización (Beck, 1998a) o como consecuencias inesperadas de la modernidad (Giddens, 1995), o, desde la perspectiva sistémica como problemas surgidos de la creciente diferenciación funcional (Luhmann 1998a; en América Latina V. Mascareño, 2000) nos parecen situaciones producto de desarrollos extremos a los que ha llegado la evolución de las comunicaciones sociales teniendo supuesta la matriz cultural moderna. Un factor que acelera dicho efecto es la mediatización de la cultura (Larraín, 2005), la que exacerba aun más la espiral de comunicaciones que refuerzan la matriz moderna y renuevan, con una rapidez alarmante, la escalada de comunicaciones que implican expresiones cada vez más extremas.

Así, detrás de problemas actuales que afectan la vida cotidiana como por ejemplo los embarazos adolescentes, el aumento del alcoholismo juvenil y la pornografía, están los desarrollos acumulativos sobre la semántica de la individualidad, su autonomía y búsqueda de bienestar y placer. El hombre moderno que dejó de ser una criatura de Dios, un ser espiritual, miembro de una comunidad, que aceptaba la vida como un tránsito a la vida eterna, pasó a constituirse en un ser individual, un sujeto frente a un mundo de objetos, y como tal ha estado persiguiendo por varios siglos cada vez mayores niveles de libertad, autonomía, bienestar y placer (Dockendorff, 2002). 
Desde nuestra perspectiva, la persistencia y extensión del neoliberalismo a nivel global es otro ejemplo en el cual reconocer la gravitación de la matriz cultural moderna. Si bien es ampliamente observado que los procesos de globalización son al mismo tiempo procesos de dominación y poder (Larraín, 2005), pensamos que más allá de ello, el neoliberalismo surge y se mantiene en la medida que es un desarrollo (una escalada de comunicaciones) que mantiene una férrea coherencia con la matriz cultural moderna. Desde esta perspectiva, lo que afecta la vida cotidiana moderna es el hecho de que el neoliberalismo ha logrado mantener y continuar imponiendo en las identidades personales la idea moderna de individuo, llevada a una expresión extrema. Es lo que se ha llamado críticamente individualismo, una especie de sacralización de la autonomía y el bienestar del individuo que afecta profundamente las relaciones sociales en todos los ámbitos, particularmente en sociedades que han aceptado sin contrapeso patrones culturales de sociedades dominantes, como es, en general, el caso de América Latina.

Como en estos ejemplos, es posible rastrear la relación con la matriz cultural moderna de gran parte de los problemas que afectan la vida cotidiana del hombre moderno. Su solución radical pasa, de esta manera, por el reemplazo de la matriz cultural moderna, lo que, sin embargo, dada la complejidad y diferenciación de la sociedad moderna, no dependerá del esfuerzo de individuos particulares sino será resultado de la propia evolución de la sociedad.

\section{Luces y sombras de la emancipación: sociología, normatividad, intervención social}

Pero el hecho de que la matriz cultural sólo llegue a cambiar producto de la evolución de la sociedad, no implica adoptar una actitud pasiva y resignada ante los problemas sociales que se originan, justamente, a partir de su vigencia y la escalada de comunicaciones que llevan sus supuestos a desarrollos extremos. Si bien es cierto que no son los individuos los que tienen en su poder decidir cuáles comunicaciones serán reestabilizadas por la sociedad, son los individuos (sistemas síquicos) los que gatillan las variaciones que pueden llegar a ser reestabilizadas y desplazar comunicaciones sedimentadas que aun mantienen su poder estructurante. Esto nos pone en el terreno de la intervención social y por ende en el de la normatividad en la sociología.

\section{Normatividad y sociología}

El tema central aquí es la disyuntiva entre una sociología que "hace visible lo que acontece de todos modos y que en modo alguno cabe modificar" (Schelsky en Habermas, 1997: 284), considerada conservadora, y una que asume la crítica, a partir de su propia tradición y que por lo tanto puede "protegerse a sí misma y a la sociedad contra un cultivo acrítico de lo existente, contra su consolidación consumada ingenuamente con los medios científicos" (Habermas, 1997: 286) Planteada la disyuntiva así, si el sociólogo no quiere terminar como un ingenuo, o si le importa participar en el destino de la sociedad, no podría sino optar por una sociología crítica.

Pero la disyuntiva no es tan simple; "modificar" puede no sólo ser crítico sin también conservador. La historia de la sociología así lo muestra, según lo expone el propio Habermas. El autor sostiene que históricamente la sociología ha sido a la vez crítica y conservadora; que desde la Revolución Francesa es ciencia de la crisis, pero crisis que 
puede ser vista sociológicamente como emancipación o como anarquía, como revolución o restauración, según hayan sido las opciones ideológicas de los sociólogos, como los casos de Saint Simon y De Bonald (Habermas, 1997).

De acuerdo a la tradición científica, a la que se adscribe la sociología, como ciencia ella debe ser neutra a sus consecuencias políticas. Sin embargo, desde el propio quehacer científico, Marx le dio la tarea crítica de incluir la praxis. Para Habermas (1997), antes de Marx la teoría guardaba la esperanza de que alguien la transformara en praxis. Habermas termina dando vuelta el sentido de lo conservador, afirmando que lo conservador de la sociología, es su crítica.

A partir de la relación entre lo científico y lo político, cabe separar en el sociólogo entre ambos roles. Aquí la disyuntiva pareciera tener fácil solución porque si el sociólogo se atiene a su doble papel como científico y como ciudadano (Habermas, 1997), puede elegir las tareas o temas que desea cultivar sociológicamente según puntos de vista de relevancia política, y separar semejante decisión -como ciudadano-, del trabajo científico mismo, cumpliendo así la expectativa de la ciencia de que como científico no debe permitir la influencia política en su quehacer.

Pero, la pregunta sobre si es posible un quehacer científico en sociología, esto es, una tarea puramente descriptiva sin dejarse contaminar por lo normativo, no se resuelve con una separación deliberada de los roles del sociólogo. Existe la convicción de que el describir conlleva siempre una agenda normativa, sólo que oculta. Desde este punto de vista, pretender una neutralidad descriptiva sería caer presa de una ingenuidad normativa. Esta perspectiva se ve reforzada cuando se asume que lo que caracteriza a lo social es, precisamente, lo normativo.

Nuestra observación es que efectivamente, y como bien se desprende del terreno apriorístico destacado por Alexander (1992) y Ritzer (1988), la neutralidad normativa es una imposibilidad; indefectiblemente se cuelan valores y preferencias que se mantienen como presupuestos tras el quehacer científico del sociólogo. Sin embargo, ello no implica que una dimensión normativa tenga que ser incluida como parte de la arquitectura teórica. Se puede seguir a Luhmann en sacar la normatividad de la sociología y mantenerse en la descripción cuando se hace teoría y -ya sin seguir a Luhmann-, introducir una normatividad, en el sentido de intención de orientación, como materia de propuesta, lo que puede considerarse o no, parte de la propia tarea del sociólogo. A nuestro juicio, una disciplina teórica libre de determinaciones normativas en tanto descripción, está mejor posicionada para adoptar una orientación y ofrecer, con afán de emancipación, propuestas de intervención social, justamente considerando que lo que caracteriza a lo social es lo normativo. Si se es ingenuo normativamente porque al describir hay una agenda normativa oculta, ello puede salvarse explicitando la "agenda normativa" propia, pero no necesariamente incluyéndola en la teoría.

Nuestra opción entonces, a diferencia de incluir lo normativo en la arquitectura de la teoría como en el caso de Habermas, por considerarla ineludible, se inclina por distinguir niveles, manteniendo la descripción al nivel de la teoría, y, en el nivel de la intervención social explicitar la perspectiva ética que nos inspira y de ese modo dar cuenta de la propia no-neutralidad normativa. 


\section{Un modelo sistémico de intervención social}

Acorde con lo anterior, nuestra construcción de una teoría de la cultura se proyecta también al campo de la intervención social, puesto que como afirmáramos, aun desde la teoría de sistemas sociales, junto a un propósito descriptivo científico puede abrigarse un afán de contribuir a enfrentar los problemas con que se auto-observa la sociedad contemporánea. No obstante las dificultades que opone la sociedad actual para aceptar intervenciones, dada su gran diferenciación, el concepto de cultura y particularmente el de matriz cultural, pueden resultar efectivas herramientas para identificar posibilidades y diseñar iniciativas de intervención social (Dockendorff, 2002).

El modelo sistémico de intervención social que proponemos, visualiza los problemas que la propia sociedad auto-identifica y que dicen relación con las expresiones extremas de la escalada histórica de comunicaciones cuyos supuestos subyacen en la matriz cultural. Metodológicamente, los problemas pueden ser observados a través de investigaciones empíricas, en particular los problemas reflejados en la auto-descripción que la sociedad comunica a través de los medios de comunicación masivos, en tanto hoy son éstos los que privilegiadamente muestran la realidad de la vida cotidiana moderna. Desde ese diagnóstico intenta poner - estratégicamente- ideas alternativas en las comunicaciones sociales.

El hecho que la perspectiva teórica adoptada no incorpore una dimensión normativa, obliga a definir una orientación para sustentar la intervención social. En la medida que tal orientación dice relación con un diagnóstico negativo y una propuesta de superación, se trata de una orientación ética. De este modo, la aplicación del modelo de intervención propuesto debe partir por explicitar la perspectiva ética específica desde la cual va a fundamentar la particular modalidad de intervención social que propone. Esta dependerá de la perspectiva ética que adopte el observador, en este caso el interventor, y puede consistir en un sustento pre-argumentativo, como la pasión por la justicia social o una "visión obsesionante de la unidad de la comunidad humana" (Dockendorff, 2005). Al adoptar este tipo de intervención social sistémicoconstructivista, la ética se mantiene en el plano de la orientación del observador, sin ser tratada como una verdad a universalizar.

Dada su forma de operar sin embargo, el modelo mismo incorpora elementos que conllevan una dimensión ética. En efecto, se trata de un acercamiento no jerárquico, no normativo ni basado en el control como estrategia de intervención. A su vez, las razones que justifican la intervención vienen de la observación de problemas detectados por los propios potenciales intervenidos, lo que se ha llamado intervención de segundo orden. Más allá de su propia orientación ética, el interventor no define a priori contenidos ni dirección alguna para la intervención, respetando la autonomía de los sistemas intervenidos. Son ellos los que serán los protagonistas del cambio, siendo el papel del interventor el de proveer información útil para orientar el cambio hacia un destino que los propios intervenidos logren percibir como válido.

En términos sistémicos se trata de una regulación de segundo orden desde la perspectiva del interventor y una auto-regulación reflexiva desde la perspectiva del intervenido. Se presenta la intervención como contingencia en el entorno del sistema a regular, esto es, se sitúan bajo los marcos del sentido del sistema sujeto a intervención, las distinciones que se pretende introducir, de modo tal que el 
intervenido comprenda desde sus propias categorías lo que la intervención quiere hacerle ver (Mascareño, 1996). En nuestra propuesta, las distinciones que se pretende introducir se fundamentan en el conocimiento del operar de la matriz cultural y en ello radica la especificidad del modelo de intervención propuesto. Por ello, una de tareas que el modelo de intervención contempla, es precisamente la comunicación de dicho conocimiento, ofrecido como marco general de intervención, de manera que pueda ser adoptado por diversos potenciales interventores.

El poner dicho conocimiento a disposición de la auto-reflexión de la sociedad puede resultar un aporte en tanto el develamiento del operar de la matriz cultural previene contra la identificación de fenómenos responsables de problemas allí donde se trata más bien de consecuencias del operar de dicha matriz. Desconectados de sus raíces culturales, fenómenos como, por ejemplo, el capitalismo o la crisis medio-ambiental adquieren connotaciones diferentes a si son vistos como desarrollos lógicos, llevados niveles extremos, de una manera de entender y comunicar el mundo.

El develamiento del operar de la matriz cultural es la primera y más general estrategia de intervención. Estrategias más específicas pretenden identificar y divulgar, bajo la lógica del modelo sistémico descrito, comunicaciones divergentes de la matriz cultural y eventualmente susceptibles de constituirse en nuevas selecciones sedimentadas, si son reestabilizadas por la sociedad. Bajo la orientación sistémica adoptada, se trata de la única manera viable de producir cambios.

En síntesis, el modelo sistémico de intervención social fundamenta su estrategia en una mirada histórica, evolutiva y cultural, y opta por una intervención de segundo orden, intentando contribuir a la auto-reflexión de la sociedad con una mirada que revela aspectos poco considerados en la crítica a la modernidad. Dicho modelo puede ofrecer asimismo, un diagnóstico de la contemporaneidad que fundamente intervenciones sociales no ideológicas y éticamente transparentes. RM

\section{Bibliografía}

ALEXANDER, J. 1992. Las teorías sociológicas desde la segunda guerra mundial. Barcelona: Gedisa.

ARCHER, M. 1996. Culture and Agency: the place of culture in social theory. Cambridge: Cambridge University Press.

_- . 2005. Tendencias de la Teoría Sociológica en el siglo XXI. Seminario dictado en Santiago de Chile. Universidades Alberto Hurtado y Pontificia Universidad Católica.

ARNOLD, M. 2004. "La construcción del conocimiento. Fundamentos epistemológicos del constructivismo sociopoiético". En Revista del Instituto de Investigaciones Histórico Sociales, Universidad Nacional Mayor de San Marcos. Año VIII / N 12 / Lima, pp. 271-289.

BAUMAN, Z. 1999. La Globalización, consecuencias humanas. Buenos Aires: Fondo de Cultura Económica.

BECK, U. 1998a. ¿Qué es la globalización? Barcelona: Ediciones Paidós 
_-. 1998b. La Sociedad del Riesgo. Barcelona: Ediciones Paidós.

CHERNILO, D. 2006. Teoría Sociológica I: estrategias de construcción teórica en sociología. Programa de curso para el doctorado en sociología, Universidad Alberto Hurtado. Chile.

CHERNILO, D. y MASCAREÑO, A. 2005. “Universalismo, Particularismo y Sociedad Mundial". En Persona y Sociedad, XIX (3), pp. 17-45.

DOCKENDORFF, C. 2002. Paradigma sociocultural: un concepto y una mirada al surgimiento, vigencia y cambio de los supuestos básicos que subyacen a la modernidad. Tesis para optar al grado de Magíster en Antropología y Desarrollo. Facultad de Ciencias Sociales, Universidad de Chile.

__. 2005. "La pasión por la justicia social: espiritualidad, reflexión sociológica y acción social en un contexto moderno." En Alberto Hurtado, Memoria y Actualidad. Ediciones Universidad Alberto Hurtado, Santiago, Chile.

_-- 2006a. “Lineamientos para una teoría sistémica de la cultura: la unidad semántica de la diferencia estructural". En Francisco Osorio y Eduardo Aguado, eds. La nueva teoría social en Hispanoamérica: introducción a la teoría de sistemas constructivista. Toluca, México: Universidad Autónoma del Estado de México.

_-_ 2006b. "Evolución de la cultura: la deriva semántica del cambio estructural". En Persona y Sociedad, XX (1), pp. 45-74.

EMMET, D. 1994. The role the unrealisable: a study in reguative ideals. New York: St. Martin's Press.

GIDDENS, A. 1993. Consecuencias de la Modernidad. Madrid: Editorial Alianza.

_-. 1995. Modernidad e Identidad del Yo. Barcelona: Editorial Península.

HABERMAS, J. 1991. Teoría de la Acción Comunicativa. Vol 2. Madrid: Taurus Humanidades.

_-_.1997. Teoría y Praxis: estudios de filosofía social. Madrid: Editorial Tecnos.

LARRAÍN, J. 2005. ¿América Latina Moderna? Globalización e Identidad. Santiago de Chile: LOM Ediciones.

LUHMANN, N. y DE GEORGI, R. 1993. Teoría de la Sociedad. México: Universidad I beroamericana.

1991. Sistemas sociales. Lineamientos para una teoría general. México D.F.: Alianza Universidad Textos.

_-. 1997. Observaciones de la Modernidad. Barcelona: Ediciones Paidós 
. 1998a. Complejidad y Modernidad: De la Unidad a la Diferencia. Madrid: Ed. Trotta.

. 1998b. "La cultura como un concepto histórico". En Luhmann, Teoría de los Sistemas Sociales II. Osorno: Universidad I beroamericana/I nstituto de Estudios Superiores de Occidente/Universidad de Los Lagos.

MASCAREÑO, A. 1996. Auto-Regulación en Sistemas Sociales Autónomos. Inédito. Santiago de Chile.

_-_. 2000. “Diferenciación funcional en América Latina: Los contornos de una sociedad concéntrica y los dilemas de su transformación". En Persona y Sociedad, Vol. XIV, abril, pp. 187-207.

__. 2007. "Sociología de la cultura: La deconstrucción de lo mapuche". En Revista de Estudios Públicos. Verano 2007.

MATURANA, H. y VARELA, F. 1984. El Árbol del Conocimiento. Santiago de Chile: Editorial Universitaria.

OUTHWAITE, W. 2006. Future of Society. Londres: Blackwell.

RITZER, G. 1988. "A defense of a subfield by a delination of its parameters." En Sociological Theory $6(2)$

SAUSSURE, F. 1969. Curso de Lingüística General, Buenos Aires: Ed. Losada.

VON FOERSTER, H. 1990. Bases Epistemológicas. Barcelona: Ed. Anthropos.

WAGNER, P. 2001. A history and theory of the social sciences. Londres: Sage.

\section{Notas}

${ }^{1}$ El partir de una distinción que realiza un sistema observador significa asumir que antes de que se establezca una distinción no "existe" nada. Aunque el lenguaje constructivista-sistémico utilice expresiones que parecieran reificar "objetos", como describir a los sistemas como dotados de memoria, no se trata de objetos sino de distinciones.

2 Coinciden en ello Chernilo y Mascareño (2005), al afirmar que las teorías universalistas, como la diferenciación funcional, la emergencia de la sociedad y el cosmopolitismo normativo, permiten superar obstáculos epistemológicos en los que han visto caer a la teoría sociológica en América Latina.

${ }^{3}$ Está claro que la constelación de selecciones semánticas estructurantes que conforman una matriz cultural son siempre contingentes, es decir podrían haber sido de otra manera, y esa misma contingencia es la que permite que surjan variaciones y la constelación de selecciones cambie en el tiempo. Por ello su descripción es siempre, además de semántica, histórica y localizada.

${ }^{4}$ Cabe enfatizar que la matriz cultural no tiene existencia institucionalizada ni roles que la encarnen a nivel de los individuos. Sus contenidos no son valores ni normas, son supuestos implícitos.

${ }^{5}$ Wagner (1997) agrega que la sociología es un intento de entender la modernidad y por lo tanto es víctima de la falta de distancia con su objeto, pero que aun así es tarea de la sociología el hacer justicia a la doble e irreductible naturaleza de la modernidad, idea que básicamente compartimos. 
${ }^{6}$ En este contexto la individualidad está considerada una semántica en tanto se tata de una representación, a diferencia de la concepción durkheimiana del individuo considerado como elemento constitutivo de la sociedad.

${ }^{7}$ La matriz cultural medieval contaba con una constelación de semánticas sedimentadas que concebían al individuo primariamente como una criatura de Dios, y en relación con la sociedad, como miembro de la comunidad orgánica, esto es, un cuerpo inviolable de costumbres e historia comunes constituyendo un espíritu colectivo. El hombre se veía a sí mismo subordinado a la comunidad, cuyos intereses le demandaban obediencia (Dockendorff, 2002). Así, por ejemplo, en la matriz cultural de la Edad Media no tenía cabida la noción de sujeto.

${ }^{8}$ Esta perspectiva no pretende desconocer sino complementar los análisis de la institucionalización histórica de la modernidad. Concordamos con Larraín cuando afirma que "toda modernización es un campo interpretativo...una lucha por institucionalizar las significaciones imaginarias de la modernidad en algún sentido determinado" (Larraín, 2005: 26) 